\title{
Solution of Nonlinear Space-Time Fractional Differential Equations Using the Fractional Riccati Expansion Method
}

\author{
Emad A.-B. Abdel-Salam ${ }^{1,2}$ and Eltayeb A. Yousif ${ }^{2,3}$ \\ ${ }^{1}$ Department of Mathematics, Faculty of Science, Assiut University, New Valley Branch, El-Kharja 72511, Egypt \\ ${ }^{2}$ Department of Mathematics, Faculty of Science, Northern Border University, Arar 91431, Saudi Arabia \\ ${ }^{3}$ Department of Applied Mathematics, Faculty of Mathematical Sciences, University of Khartoum, 11111 Khartoum, Sudan
}

Correspondence should be addressed to Emad A.-B. Abdel-Salam; emad_abdelsalam@yahoo.com

Received 19 June 2013; Accepted 2 December 2013

Academic Editor: Andrzej Swierniak

Copyright (C) 2013 E.-B. Abdel-Salam and E. A. Yousif. This is an open access article distributed under the Creative Commons Attribution License, which permits unrestricted use, distribution, and reproduction in any medium, provided the original work is properly cited.

\begin{abstract}
The fractional Riccati expansion method is proposed to solve fractional differential equations. To illustrate the effectiveness of the method, space-time fractional Korteweg-de Vries equation, regularized long-wave equation, Boussinesq equation, and KleinGordon equation are considered. As a result, abundant types of exact analytical solutions are obtained. These solutions include generalized trigonometric and hyperbolic functions solutions which may be useful for further understanding of the mechanisms of the complicated nonlinear physical phenomena and fractional differential equations. Among these solutions, some are found for the first time. The periodic and kink solutions are founded as special case.
\end{abstract}

\section{Introduction}

During recent years, fractional differential equations (FDEs) have attracted much attention due to their numerous applications in areas of physics, biology, and engineering [1-3]. Many important phenomena in non-Brownian motion, signal processing, systems identification, control problem, viscoelastic materials, polymers, and other areas of science are well described by fractional differential equation [4-7]. The most important advantage of using FDEs is their nonlocal property, which means that the next state of a system depends not only upon its current state but also upon all of its historical states $[8,9]$. Recently, the fractional functional analysis has been investigated by many researchers $[10,11]$. For example, the properties and theorems of Yang-Laplace transforms and Yang-Fourier transforms [12] and their applications to the fractional ordinary differential equations, fractional ordinary differential systems, and fractional partial differential equations have been discussed. Many powerful methods have been established and developed to obtain numerical and analytical solutions of FDEs, such as finite difference method [13], finite element method [14], Adomian decomposition method
$[15,16]$, differential transform method [17], variational iteration method [18-20], homotopy perturbation method [21, 22], the fractional sub-equation method [23], and generalized fractional subequation method [24]. How to extend the existing methods to solve other FDEs is still an interesting and important research problem. Thanks to the efforts of many researchers, several FDEs have been investigated and solved, such as the impulsive fractional differential equations [25], space- and time-fractional advection-dispersion equation [26-28], fractional generalized Burgers' fluid [29], and fractional heat- and wave-like equations [30], and so forth. The finding of a new mathematical algorithm to construct exact solutions of nonlinear FDEs is important and might have significant impact on future research. In this research paper, we introduce the fractional Riccati expansion method to construct many exact traveling wave solutions of nonlinear FDEs with the modified Riemann-Liouville derivative defined by Jumarie. We use the fractional Riccati expansion method for solving the space-time fractional Korteweg-de Vries (KdV) equation, space-time fractional regularized long-wave (RLW) equation, space-time fractional Boussinesq equation, and space-time fractional Klein-Gordon equation. 
The structure of this paper is as follows: in Section 2, we introduce some basic definitions and mathematical preliminaries of the fractional calculus theory. Section 3 describes the fractional Riccati expansion method for solving nonlinear FDEs. In Section 4, we applied the proposed method to the space-time fractional KdV, RLW, Boussinesq, and Klein-Gordon equations. Finally, we give some conclusions and discussions.

\section{Mathematical Preliminaries}

Fractional calculus is one of the generalizations of ordinary calculus. Generally speaking, there are two kinds of fractional derivatives. One of them is nonlocal fractional derivative, that is, Caputo derivative and Riemann-Liouville derivative which have been used successfully in various fields of science and engineering. However, the Caputo derivative requires the function to be smooth and differentiable. Obviously, the nonlocal derivatives are not suitable for the investigation of the local behavior of fractional differentiable equations. The other one is the local fractional derivative, that is, KolwankarGangal (K-G) derivative [31], Chen's fractal derivative [32] and Cresson's derivative [33]. One of the famous examples is the devi-stair curve, which can be described by a continuous but nowhere differentiable function. Recently, there is new development of continuous but nowhere differentiable functions [34]. This study is motivated by the need to propose a fractional Riccati expansion method to construct exact analytical solutions of nonlinear FDEs with the following modified Riemann-Liouville derivative defined by Jumarie [35]:

$$
\begin{aligned}
& D_{x}^{\alpha} f(x) \\
& = \begin{cases}\frac{1}{\Gamma(1-\alpha)} \int_{0}^{x}(x-\xi)^{-\alpha-1}[f(\xi)-f(0)] d \xi, & \alpha<0, \\
\frac{1}{\Gamma(1-\alpha)} \frac{d}{d x} \int_{0}^{x}(x-\xi)^{-\alpha}[f(\xi)-f(0)] d \xi, & 0<\alpha<1, \\
{\left[f^{(\alpha-n)}(x)\right]^{(n)},} & n \leq \alpha<n+1, n \geq 1,\end{cases}
\end{aligned}
$$

which has merits over the original one; for example, the $\alpha$-order derivative of a constant is zero. Owing to these merits, Jumarie's modified Riemann-Liouville derivative was successfully applied to the probability calculus [36], fractional Laplace problems [37], and fractional variational calculus [38]. Some useful formulas and results of Jumarie's modified Riemann-Liouville derivative were summarized in [35], three of them are

$$
\begin{gathered}
D_{x}^{\alpha} x^{\gamma}=\frac{\Gamma(\gamma+1)}{\Gamma(\gamma+1-\alpha)} x^{\gamma-\alpha}, \quad \gamma>0, \\
D_{x}^{\alpha}[f(x) g(x)]=g(x) D_{x}^{\alpha} f(x)+f(x) D_{x}^{\alpha} g(x), \\
D_{x}^{\alpha} f[g(x)]=f_{g}^{\prime}[g(x)] D_{x}^{\alpha} g(x)=D_{g}^{\alpha} f[g(x)]\left(g_{x}^{\prime}\right)^{\alpha},
\end{gathered}
$$

which will be used in the following sections.

\section{Fractional Riccati Expansion Method}

In this section, we outline the main steps of the fractional Riccati expansion method for solving nonlinear FDEs. For a given nonlinear FDE, say, in two variables $x$ and $t$

$$
P\left(u, D_{t}^{\alpha} u, D_{x}^{\alpha} u, D_{t}^{2 \alpha} u, D_{x}^{2 \alpha} u, \ldots\right)=0
$$

where $D_{t}^{\alpha} u$ and $D_{x}^{\alpha} u$ are Jumarie's modified RiemannLiouville derivatives of $u, u=u(x, t)$ is an unknown function, and $P$ is a polynomial in $u$ and its various partial derivatives, in which the highest order derivatives and nonlinear terms are involved.

Step 1. By using the traveling wave transformation:

$$
u(x, t)=u(\xi), \quad \xi=x+\omega t,
$$

where $\omega$ is a constant to be determined later, the nonlinear FDE (3) is reduced to the following nonlinear fractional ordinary differential equation (FODE) for $u=u(\xi)$ :

$$
\widetilde{P}\left(u, \omega^{\alpha} D_{\xi}^{\alpha} u, D_{\xi}^{\alpha} u, \omega^{2 \alpha} D_{\xi}^{2 \alpha} u, D_{\xi}^{2 \alpha} u, \ldots\right)=0 .
$$

Step 2. We suppose that $u(\xi)$ can be expressed by a finite power series of $F(\xi)$ as follows:

$$
u(\xi)=a_{0}+\sum_{i=1}^{n} a_{i} F^{i}, \quad a_{n} \neq 0,
$$

where $a_{i}(i=0,1,2, \ldots, n)$ are constants to be determined later, $n$ is a positive integer determined by balancing the linear term of the highest order with the nonlinear term in (5), and $F=F(\xi)$ satisfies the following fractional Riccati equation:

$$
D_{\xi}^{\alpha} F=A+B F^{2}, \quad 0<\alpha \leq 1,
$$

where $A$ and $B$ are constants. Using the Mittag-Leffler function in one parameter $E_{\alpha}(x)=\sum_{k=0}^{\infty}\left(x^{k} / \Gamma(1+k \alpha)\right)(\alpha>0)$, we obtain the following solution of (7).

Case 1. If $A=1$ and $B=1$, then (7) has the solution $F=$ $\tan (\xi, \alpha)$.

Case 2. If $A=-1$ and $B=-1$, then (7) has the solution $F=$ $\cot (\xi, \alpha)$.

Case 3. If $A=1$ and $B=-1$, then (7) has the solutions

$$
F=\tanh (\xi, \alpha), \quad F=\operatorname{coth}(\xi, \alpha) .
$$

Case 4. If $A=1 / 2$ and $B=-1 / 2$, then (7) has the solutions

$$
F=\frac{\tanh (\xi, \alpha)}{1 \pm \operatorname{sech}(\xi, \alpha)}, \quad F=\operatorname{coth}(\xi, \alpha) \pm \operatorname{csch}(\xi, \alpha) \text {. }
$$

Case 5. If $A=1 / 2$ and $B=1 / 2$, then (7) has the solutions

$$
\begin{gathered}
F=\frac{\tan (\xi, \alpha)}{1 \pm \sec (\xi, \alpha)}, \quad F=\csc (\xi, \alpha)-\cot (\xi, \alpha), \\
F=\tan (\xi, \alpha) \pm \sec (\xi, \alpha) .
\end{gathered}
$$


Case 6. If $A=-1 / 2$ and $B=-1 / 2$, then (7) has the solutions

$$
\begin{gathered}
F=\frac{\cot (\xi, \alpha)}{1 \pm \csc (\xi, \alpha)}, \\
F=\sec (\xi, \alpha)-\tan (\xi, \alpha), \\
F=\cot (\xi, \alpha) \pm \csc (\xi, \alpha) .
\end{gathered}
$$

Case 7. If $A=1$ and $B=-4$, then (7) has the solution $F=$ $(\tanh (\xi, \alpha)) /\left(1+\tanh ^{2}(\xi, \alpha)\right)$.

Case 8. If $A=1$ and $B=4$, then (7) has the solution $F=$ $(\tan (\xi, \alpha)) /\left(1-\tan ^{2}(\xi, \alpha)\right)$.

Case 9. If $A=-1$ and $B=-4$, then (7) has the solution $F=$ $(\cot (\xi, \alpha)) /\left(1-\cot ^{2}(\xi, \alpha)\right)$, where the generalized hyperbolic and trigonometric functions are defined as

$$
\begin{gathered}
\cosh (\xi, \alpha)=\frac{E_{\alpha}\left(\xi^{\alpha}\right)+E_{\alpha}\left(-\xi^{\alpha}\right)}{2}, \\
\sinh (\xi, \alpha)=\frac{E_{\alpha}\left(\xi^{\alpha}\right)-E_{\alpha}\left(-\xi^{\alpha}\right)}{2}, \\
\cos (\xi, \alpha)=\frac{E_{\alpha}\left(i \xi^{\alpha}\right)+E_{\alpha}\left(-i \xi^{\alpha}\right)}{2}, \\
\sin (\xi, \alpha)=\frac{E_{\alpha}\left(i \xi^{\alpha}\right)-E_{\alpha}\left(-i \xi^{\alpha}\right)}{2 i}, \\
\tanh (\xi, \alpha)=\frac{\sinh (\xi, \alpha)}{\cosh (\xi, \alpha)}, \quad \tan (\xi, \alpha)=\frac{\sin (\xi, \alpha)}{\cos (\xi, \alpha)}, \\
\operatorname{coth}(\xi, \alpha)=\frac{1}{\tanh (\xi, \alpha)}, \quad \operatorname{sech}(\xi, \alpha)=\frac{1}{\cosh (\xi, \alpha)}, \\
\cot (\xi, \alpha)=\frac{1}{\tan (\xi, \alpha)}, \quad \sec (\xi, \alpha)=\frac{1}{\cos (\xi, \alpha)}, \\
\operatorname{csch}(\xi, \alpha)=\frac{1}{\sinh (\xi, \alpha)}, \quad \csc (\xi, \alpha)=\frac{1}{\sin (\xi, \alpha)} .
\end{gathered}
$$

Step 3. After substituting the fractional Riccati expansion method (6) into the FODE (5) the left-hand side of (5) can be converted into a polynomial in $F(\xi)$. Setting each coefficient of the polynomial to zero yields system of algebraic equations for $a_{0}, a_{1}, \ldots, a_{n}$, and $\omega$.

Step 4. By solving the system obtained in Step 3, the constants $a_{0}, a_{1}, \ldots, a_{n}$, and $\omega$ can be expressed by the parameters $A$ and $B$. Depending on the chosen values of $A$ and $B$ the function $F(\xi)$ possesses the traveling wave solutions as given above; then the fractional Riccati expansion method (6) has the traveling wave solution of the nonlinear FDE (3).

Remark 1. If we take $A=\sigma$ and $B=1$, this agrees with the results obtained by S. Zhang and H. Q. Zhang [23].

Remark 2. In [39], Professor He introduced the simple fractional complex transform to convert nonlinear FDEs into its nonlinear differential equations.

\section{Application}

4.1. Space-Time Fractional KdV Equation. The KdV equation is the earliest soliton equation that was firstly derived by Korteweg and de Vries to model the evolution of shallow water wave in 1895 . The KdV-type equations have applications in shallow-water waves [40], optical solitons in the two cycle regime [41], density waves in traffic flow of two kinds of vehicles [42], short waves in nonlinear dispersive models [43], surface acoustic soliton in a system supporting long waves [44], quantum field theory, plasma physics, and solid-state physics. The space-time fractional $\mathrm{KdV}$ equation is

$$
D_{t}^{\alpha} u+\mu u D_{x}^{\alpha} u+\tau D_{x}^{3 \alpha} u=0, \quad 0<\alpha \leq 1,
$$

where $\mu$ and $\tau$ are constants. In order to solve (13) by the fractional Riccati expansion method, we use the travelling wave transformation $u(x, t)=u(\xi), \xi=x+\omega t$, where $\omega$ is the dimensionless velocity of the wave. Then, (13) is reduced to the following nonlinear FODE:

$$
\omega^{\alpha} D_{\xi}^{\alpha} u+\mu u D_{\xi}^{\alpha} u+\tau D_{\xi}^{3 \alpha} u=0 .
$$

Balancing $D_{\xi}^{3 \alpha} u$ with $u D_{\xi}^{\alpha} u$ gives $n=2$. Therefore, the solution of (14) can be expressed as

$$
u=a_{0}+a_{1} F+a_{2} F^{2} .
$$

By substituting (15) into (14) using (7) and setting the coefficients of $F^{i}(i=0,1,2,3,4,5)$ to zero, we get

$$
a_{0}=-\frac{\omega^{\alpha}+8 \tau A B}{\mu}, \quad a_{1}=0, \quad a_{2}=-\frac{12 \tau B^{2}}{\mu} .
$$

The general formula for the traveling wave solution of the space-time fractional $\mathrm{KdV}$ equation (13)

$$
u(x, t)=-\frac{1}{\mu}\left[\omega^{\alpha}+8 \tau A B+12 \tau B^{2} F^{2}(x+\omega t)\right] .
$$

By selecting the special value of the $A, B$, and the corresponding function $F(\xi)$, we get the following solutions of (13):

$$
\begin{aligned}
& u_{1}=-\frac{1}{\mu}\left[\omega^{\alpha}+8 \tau+12 \tau \tan ^{2}(x+\omega t, \alpha)\right], \\
& u_{2}=-\frac{1}{\mu}\left[\omega^{\alpha}-8 \tau+12 \tau \tanh ^{2}(x+\omega t, \alpha)\right] .
\end{aligned}
$$

The remaining solutions can be obtained in a similar manner. When $\alpha=1$, we obtain the classical KdV equation

$$
u_{t}+\mu u u_{x}+\tau u_{x x x}=0,
$$

as special case of (13). Solutions given in (18) reduced to the well-known periodic and kink solutions of the KdV equation

$$
\begin{aligned}
& u_{1 \mathrm{KdV}}=-\frac{1}{\mu}\left[\omega+8 \tau+12 \tau \tan ^{2}(x+\omega t)\right], \\
& u_{2 \mathrm{KdV}}=-\frac{1}{\mu}\left[\omega-8 \tau+12 \tau \tanh ^{2}(x+\omega t)\right] .
\end{aligned}
$$


4.2. Space-Time Fractional Regularized Long-Wave Equation. The RLW equation that describes approximately the unidirectional propagation of long waves in certain nonlinear dispersive systems has been proposed by Benjamin et al. in 1972. The RLW equation is considered as an alternative to the $\mathrm{KdV}$ equation, which is modeled to govern a large number of physical phenomena such as shallow waters and plasma waves [45]. The space-time fractional RLW equation has the form

$$
D_{t}^{\alpha} u+\nu D_{x}^{\alpha} u+\mu u D_{x}^{\alpha} u-\tau D_{t}^{\alpha} D_{x}^{2 \alpha} u=0, \quad 0<\alpha \leq 1,
$$

where $\nu, \mu$, and $\tau$ are constants. By using the travelling wave transformation $u(x, t)=u(\xi), \xi=x+\omega t,(21)$ is reduced to the following nonlinear FODE:

$$
\left(\omega^{\alpha}+\nu\right) D_{\xi}^{\alpha} u+\mu u D_{\xi}^{\alpha} u-\tau \omega^{\alpha} D_{\xi}^{3 \alpha} u=0 .
$$

Thus, the solution of (22) has the form

$$
u=a_{0}+a_{1} F+a_{2} F^{2} .
$$

By substituting (23) into (22) using (7) and setting the coefficients of $F^{i}(i=0,1,2,3,4,5)$ to zero, we have

$$
a_{0}=-\frac{\omega^{\alpha}-8 \tau A B \omega^{\alpha}+\nu}{\mu}, \quad a_{1}=0, \quad a_{2}=\frac{12 \tau B^{2} \omega^{\alpha}}{\mu} \text {. }
$$

The general formula of the travelling wave solution of the space-time fractional RLW equation (21) is

$$
u(x, t)=-\frac{1}{\mu}\left[\omega^{\alpha}-8 \tau A B \omega^{\alpha}+v-12 \tau B^{2} \omega^{\alpha} F^{2}(x+\omega t)\right] .
$$

By selecting the special value of the $A, B$, and the corresponding function $F(\xi)$, we get the following solutions of (21):

$$
\begin{aligned}
& u_{1}=-\frac{1}{\mu}\left[\omega^{\alpha}-8 \tau \omega^{\alpha}+v-12 \tau \omega^{\alpha} \tan ^{2}(x+\omega t, \alpha)\right] \\
& u_{2}=-\frac{1}{\mu}\left[\omega^{\alpha}+8 \tau \omega^{\alpha}+v-12 \tau \omega^{\alpha} \tanh ^{2}(x+\omega t, \alpha)\right] .
\end{aligned}
$$

The remaining solutions can be obtained in a similar manner. As a special case, when $\alpha=1$ (21) reduced to the classical RLW equation

$$
u_{t}+v u_{x}+\mu u u_{x}-\tau u_{x x t}=0
$$

The solutions (26) take the form of the well-known periodic and kink solutions of the RLW equation

$$
\begin{aligned}
& u_{1 \mathrm{RLW}}=-\frac{1}{\mu}\left[\omega-8 \tau \omega+\nu-12 \tau \omega \tan ^{2}(x+\omega t)\right], \\
& u_{2 \mathrm{RLW}}=-\frac{1}{\mu}\left[\omega+8 \tau \omega+\nu-12 \tau \omega \tanh ^{2}(x+\omega t)\right] .
\end{aligned}
$$

4.3. Space-Time Fractional Boussinesq Equation. The Boussinesq equation was first derived to describe the propagation of long waves in shallow water [46]. It also arises in many other applications of physical interest including one-dimensional nonlinear lattice waves, vibrations in a nonlinear string, and ion sound waves in a plasma $[47,48]$. Space-time fractional Boussinesq equation has the form

$$
D_{t}^{2 \alpha} u-v D_{x}^{2 \alpha} u-\mu D_{x}^{2 \alpha}\left(u^{2}\right)-\tau D_{x}^{4 \alpha} u=0, \quad 0<\alpha \leq 1,
$$

where $\nu, \mu$, and $\tau$ are constants. By using the travelling wave transformation $u(x, t)=u(\xi), \xi=x+\omega t,(29)$ is reduced to the following nonlinear FODE:

$$
\left(\omega^{2 \alpha}-\nu\right) D_{\xi}^{2 \alpha} u-2 \mu\left[\left(D_{\xi}^{\alpha} u\right)^{2}+u D_{\xi}^{2 \alpha} u\right]-\tau D_{\xi}^{4 \alpha} u=0 .
$$

Thus, the solution of (30) has the form

$$
u=a_{0}+a_{1} F+a_{2} F^{2} .
$$

By substituting (31) into (30) using (7) and setting the coefficients of $F^{i}(i=0,1, \ldots, 6)$ to zero, we have

$$
a_{0}=-\frac{\omega^{2 \alpha}-8 \tau A B-\nu}{2 \mu}, \quad a_{1}=0, \quad a_{2}=-\frac{6 \tau B^{2}}{\mu} .
$$

The general formula of the travelling wave solution of the fractional space-time Boussinesq equation (29) is

$$
u(x, t)=-\frac{1}{2 \mu}\left[\omega^{2 \alpha}-8 \tau A B-\nu+12 \tau B^{2} F^{2}(x+\omega t)\right] .
$$

By selecting the special value of the $A, B$, and the corresponding function $F(\xi)$, we get the following solutions of (29):

$$
\begin{aligned}
& u_{1}=-\frac{1}{2 \mu}\left[\omega^{2 \alpha}-8 \tau-\nu+12 \tau \tan ^{2}(x+\omega t, \alpha)\right], \\
& u_{2}=-\frac{1}{2 \mu}\left[\omega^{2 \alpha}+8 \tau-\nu+12 \tau \tanh ^{2}(x+\omega t, \alpha)\right] .
\end{aligned}
$$

The remaining solutions can be obtained in a similar manner. When $\alpha=1$, we obtain the Boussinesq equation

$$
u_{t t}-v u_{x x}+\mu\left(u^{2}\right)_{x x}-\tau u_{x x x x}=0 .
$$

The solutions (34) reduced to the famous periodic and kink solutions of the Boussinesq equation

$$
\begin{aligned}
& u_{1 \text { Boussinesq }}=-\frac{1}{2 \mu}\left[\omega^{2}-8 \tau-\nu+12 \tau \tan ^{2}(x+\omega t)\right], \\
& u_{2 \text { Boussinesq }}=-\frac{1}{2 \mu}\left[\omega^{2}+8 \tau-\nu+12 \tau \tanh ^{2}(x+\omega t)\right] .
\end{aligned}
$$


4.4. Space-Time Fractional Klein-Gordon Equation. The nonlinear Klein-Gordon equation is a good physical equation in the sense that it appears in many fields of applications [49]. For example, in relativistic quantum mechanics, it describes the processes involving particles of spin zero. The nonlinear space-time fractional Klein-Gordon equation is

$$
D_{t}^{2 \alpha} u-v D_{x}^{2 \alpha} u+\mu u-\tau u^{3}=0, \quad 0<\alpha \leq 1,
$$

where $\nu, \mu$, and $\tau$ are constants. Let $u(x, t)=u(\xi), \xi=x+\omega t$, (37) transforms to the reduced nonlinear FODE

$$
\left(\omega^{2 \alpha}-v\right) D_{\xi}^{2 \alpha} u+\mu u-\tau u^{3}=0 .
$$

Balancing $D_{\xi}^{2 \alpha} u$ with $u^{3}$ yields $n=1$, so we may choose

$$
u=a_{0}+a_{1} F
$$

By substituting (39) into (38) using (7) and setting the coefficients of $F^{i}(i=0,1,2,3)$ to zero, we have

$$
a_{1}^{2}=-\frac{B \mu}{A \tau}, \quad a_{0}=0, \quad \omega^{2 \alpha}=v-\frac{\mu}{2 A B} .
$$

The general formula of the travelling wave solution of the fractional space-time Klein-Gordon equation (37) is

$$
u(x, t)= \pm \sqrt{-\frac{B \mu}{A \tau}} F(x+\omega t), \quad \omega^{2 \alpha}=v-\frac{\mu}{2 A B} .
$$

By selecting the special value of the $A, B$, and the corresponding function $F(\xi)$, we get the following solutions of (37):

$$
\begin{array}{ll}
u(x, t)= \pm \sqrt{-\frac{\mu}{\tau}} \tan (x+\omega t, \alpha), & \omega^{2 \alpha}=v-\frac{\mu}{2}, \quad \mu<0 . \\
u(x, t)= \pm \sqrt{\frac{\mu}{\tau}} \tanh (x+\omega t, \alpha), & \omega^{2 \alpha}=v-\frac{\mu}{2}, \quad \mu>0 .
\end{array}
$$

The remaining solutions can be obtained in a similar manner. When $\alpha=1$ (37) reduced to the well-known Klein-Gordon equation

$$
u_{t t}-v u_{x x}+\mu u-\tau u^{3}=0
$$

The solutions (42) take the form of periodic and kink solutions of the Klein-Gordon equation

$$
\begin{array}{ll}
u_{1 \mathrm{KG}}= \pm \sqrt{-\frac{\mu}{\tau}} \tan (x+\omega t), & \omega^{2}=v-\frac{\mu}{2}, \quad \mu<0, \\
u_{2 \mathrm{KG}}= \pm \sqrt{\frac{\mu}{\tau}} \tanh (x+\omega t), \quad \omega^{2}=v-\frac{\mu}{2}, \quad \mu>0 .
\end{array}
$$

\section{Conclusions and Discussions}

We have proposed the fractional Riccati expansion method to solve nonlinear FDEs. The space-time fractional KdV equation, RLW equation, Boussinesq equation, and Klein-Gordon equation are selected to test the effectiveness of the proposed method. As a result, some exact analytical solutions are obtained including the generalized hyperbolic function and generalized trigonometric function solutions. To the best of our knowledge, some of the solutions obtained in this research paper have not been reported in the literature. The fractional Riccati expansion method is more effective and simpler than other methods, and a number of solutions can be obtained simultaneously. Mathematical packages can be used to perform more complicated and tedious algebraic calculations. The fractional Riccati expansion method can be applied to other nonlinear FDEs. How to extend other methods used for solving differential equations, such as F-expansion method, Fan sub-equation method, auxiliary sub-equation method, and the projective Riccati equation method, to handle FDEs is worthy of study. This is our task in the future. We hope that the present solutions may be useful in further numerical analysis and may help one to explain some physical phenomena. This paper is merely an initial work; more applications to the other nonlinear physical systems could be conducted and deserve further investigation.

\section{Acknowledgment}

The authors would like to express their sincere thanks to the referees for their valuable suggestions and comments.

\section{References}

[1] A. A. Kilbas, H. M. Srivastava, and J. J. Trujillo, Theory and Applications of Fractional Differential Equations, Elsevier, Amsterdam, The Netherlands, 2006.

[2] R. Hilfer, Applications of Fractional Calculus in Physics, World Scientific, River Edge, NJ, USA, 2000.

[3] B. J. West, M. Bolognab, and P. Grigolini, Physics of Fractal Operators, Springer, New York, NY, USA, 2003.

[4] K. S. Miller and B. Ross, An Introduction to the Fractional Calculus and Fractional Differential Equations, John Wiley \& Sons, New York, NY, USA, 1993.

[5] S. G. Samko, A. A. Kilbas, and O. I. Marichev, Fractional Integrals and Derivatives: Theory and Applications, Gordon and Breach, Yverdon, Switzerland, 1993.

[6] I. Podlubny, Fractional Differential Equations, Academic Press, San Diego, Calif, USA, 1999.

[7] C. Li, A. Chen, and J. Ye, "Numerical approaches to fractional calculus and fractional ordinary differential equation," Journal of Computational Physics, vol. 230, pp. 3352-3368, 2011.

[8] Z. Odibat and S. Momani, "The variational iteration method: an efficient scheme for handling fractional partial differential equations in fluid mechanics," Computers and Mathematics with Applications, vol. 58, no. 11-12, pp. 2199-2208, 2009.

[9] J. H. He, "Some applications of nonlinear fractional differential equations and their approximations," Bulletin of Science, Technology \& Society, vol. 15, no. 2, pp. 86-90, 1999.

[10] K. Diethelm, The Analysis of Fractional Differential Equations, Springer, Berlin, Germany, 2010.

[11] I. Podlubny, Fractional Differential Equations, Academic Press, San Diego, Calif, USA, 1999.

[12] X. Yang, "Local fractional integral transforms," Progress in Nonlinear Science, vol. 4, pp. 1-225, 2011. 
[13] M. Cui, "Compact finite difference method for the fractional diffusion equation," Journal of Computational Physics, vol. 228, no. 20, pp. 7792-7804, 2009.

[14] Q. Huang, G. Huang, and H. Zhan, "A finite element solution for the fractional advection-dispersion equation," Advances in Water Resources, vol. 31, no. 12, pp. 1578-1589, 2008.

[15] A. M. A. El-Sayed and M. Gaber, “The Adomian decomposition method for solving partial differential equations of fractal order in finite domains," Physics Letters A, vol. 359, pp. 175-182, 2006.

[16] A. M. A. El-Sayed, S. H. Behiry, and W. E. Raslan, "Adomian's decomposition method for solving an intermediate fractional advection-dispersion equation," Computers and Mathematics with Applications, vol. 59, no. 5, pp. 1759-1765, 2010.

[17] Z. Odibat and S. Momani, "A generalized differential transform method for linear partial differential equations of fractional order," Applied Mathematics Letters, vol. 21, pp. 194-199, 2008.

[18] J. H. He, "Variational iteration method for delay differential equations," Communications in Nonlinear Science and Numerical Simulation, vol. 2, pp. 235-236, 1997.

[19] G. Wu and E. W. M. Lee, "Fractional variational iteration method and its application," Physics Letters A, vol. 374, pp. 2506-2509, 2010.

[20] S. Guo and L. Mei, "The fractional variational iteration method using He’s polynomials," Physics Letters A, vol. 375, pp. 309-313, 2011.

[21] J. H. He, "Homotopy perturbation technique," Computer Methods in Applied Mechanics and Engineering, vol. 178, pp. 257-262, 1999.

[22] J. H. He, "A coupling method of a homotopy technique and a perturbation technique for non-linear problems," International Journal of Non-Linear Mechanics, vol. 35, pp. 37-43, 2000.

[23] S. Zhang and H. Q. Zhang, "Fractional sub-equation method and its applications to nonlinear fractional PDEs," Physics Letters A, vol. 375, pp. 1069-1073, 2011.

[24] S. Guo, L. Mei, Y. Li, and Y. Sun, "The improved fractional subequation method and its applications to the space-time fractional differential equations in fluid mechanics," Physics Letters A, vol. 376, pp. 407-411, 2012.

[25] G. M. Mophou, "Existence and uniqueness of mild solutions to impulsive fractional differential equations," Nonlinear Analysis, Theory, Methods and Applications, vol. 72, no. 3-4, pp. 1604-1615, 2010.

[26] Q. Huang, G. Huang, and H. Zhan, "A finite element solution for the fractional advection-dispersion equation," Advances in Water Resources, vol. 31, pp. 1578-1589, 2008.

[27] W. Jiang and Y. Lin, "Approximate solution of the fractional advection-dispersion equation," Computer Physics Communications, vol. 181, pp. 557-561, 2010.

[28] R. K. Pandey, O. P. Singh, and V. K. Baranwal, "An analytic algorithm for the space-time fractional advection-dispersion equation," Computer Physics Communications, vol. 182, no. 5, pp. 1134-1144, 2011.

[29] C. Xue, J. Nie, and W. Tan, "An exact solution of start-up flow for the fractional generalized Burgers' fluid in a porous half-space," Nonlinear Analysis, Theory, Methods and Applications, vol. 69, no. 7, pp. 2086-2094, 2008.

[30] Y. Molliq R, M. S. M. Noorani, and I. Hashim, "Variational iteration method for fractional heat- and wave-like equations," Nonlinear Analysis; Real World Applications, vol. 10, no. 3, pp. 1854-1869, 2009.
[31] K. M. Kolwankar and A. D. Gangal, "Local fractional FokkerPlanck equation," Physical Review Letters, vol. 80, no. 2, pp. 214217, 1998.

[32] Y. Chen, Y. Yan, and K. Zhang, "On the local fractional derivative," Journal of Mathematical Analysis and Applications, vol. 362, no. 1, pp. 17-33, 2010.

[33] W. Chen and H. G. Sun, "Multiscale statistical model of fullydeveloped turbulence particle accelerations," Modern Physics Letters B, vol. 23, p. 449, 2009.

[34] J. Cresson, "Scale calculus and the Schrödinger equation," Journal of Mathematical Physics, vol. 44, no. 11, pp. 4907-4938, 2003.

[35] F. Ben Adda and J. Cresson, "About non-differentiable functions," Journal of Mathematical Analysis and Applications, vol. 263, no. 2, pp. 721-737, 2001.

[36] G. Jumarie, "Modified Riemann-Liouville derivative and fractional Taylor series of nondifferentiable functions further results," Computers \& Mathematics with Applications, vol. 51, no. 9-10, pp. 1367-1376, 2006.

[37] G. Jumarie, "New stochastic fractional models for Malthusian growth, the Poissonian birth process and optimal management of populations," Mathematical and Computer Modelling, vol. 44, no. 3-4, pp. 231-254, 2006.

[38] G. Jumarie, "Laplace's transform of fractional order via the Mittag-Leffler function and modified Riemann-Liouville derivative," Applied Mathematics Letters, vol. 22, no. 11, pp. 1659-1664, 2009.

[39] J.-H. He, "A short remark on fractional variational iteration method," Physics Letters A, vol. 375, no. 38, pp. 3362-3364, 2011.

[40] V. I. Karpman, "Radiation by weakly nonlinear shallow-water solitons due to higher-order dispersion," Physical Review E, vol. 58, no. 4, pp. 5070-5080, 1998.

[41] H. Leblond and F. Sanchez, "Models for optical solitons in the two-cycle regime," Physical Review A, vol. 67, no. 1, Article ID 013804, 8 pages, 2003.

[42] Z. Z. Liu, X. J. Zhou, X. M. Liu, and J. Luo, "Density waves in traffic flow of two kinds of vehicles," Physical Review E, vol. 67, no. 1, part 2, Article ID 017601, 2003.

[43] M. A. Manna and V. Merle, "Asymptotic dynamics of short waves in nonlinear dispersive models," Physical Review E, vol. 57, no. 5, pp. 6206-6209, 1998.

[44] T. Sakuma and N. Nishiguchi, "Theory of the surface acoustic soliton. V. Approximate soliton solution of the Korteweg de Vries type," Physical Review B, vol. 41, no. 17, pp. 12117-12121, 1990.

[45] T. B. Benjamin, J. L. Bona, and J. J. Mahony, "Model equations for long waves in nonlinear dispersive systems," Philosophical Transactions of the Royal Society of London A, vol. 272, no. 1220, pp. 47-78, 1972.

[46] R. M. Miura, "An inverse scattering method," Communications on Pure and Applied Mathematics, vol. 21, pp. 467-490, 1974.

[47] P. A. Clarkson and M. D. Kruskal, "New similarity reductions of the Boussinesq equation," Journal of Mathematical Physics, vol. 30, no. 10, pp. 2201-2213, 1989.

[48] M. Toda, "Studies of a non-linear lattice," Physics Reports, vol. 18, no. 1, pp. 1-123, 1975.

[49] R. F. Dashen, B. Hasslacher, and A. Neveu, "Nonperturbative methods and extended-hadron models in field theory. II. Twodimensional models and extended hadrons," Physical Review D, vol. 10, no. 12, pp. 4130-4138, 1974. 


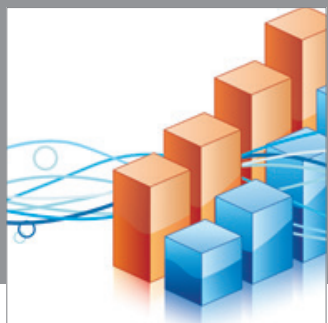

Advances in

Operations Research

mansans

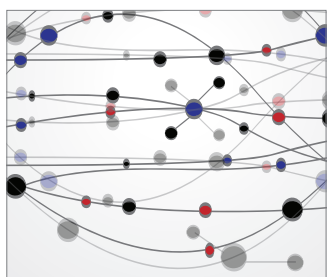

The Scientific World Journal
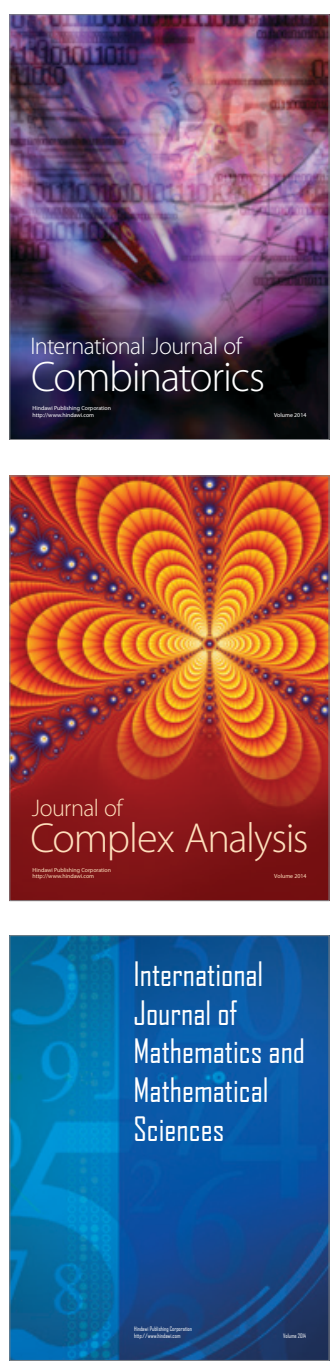
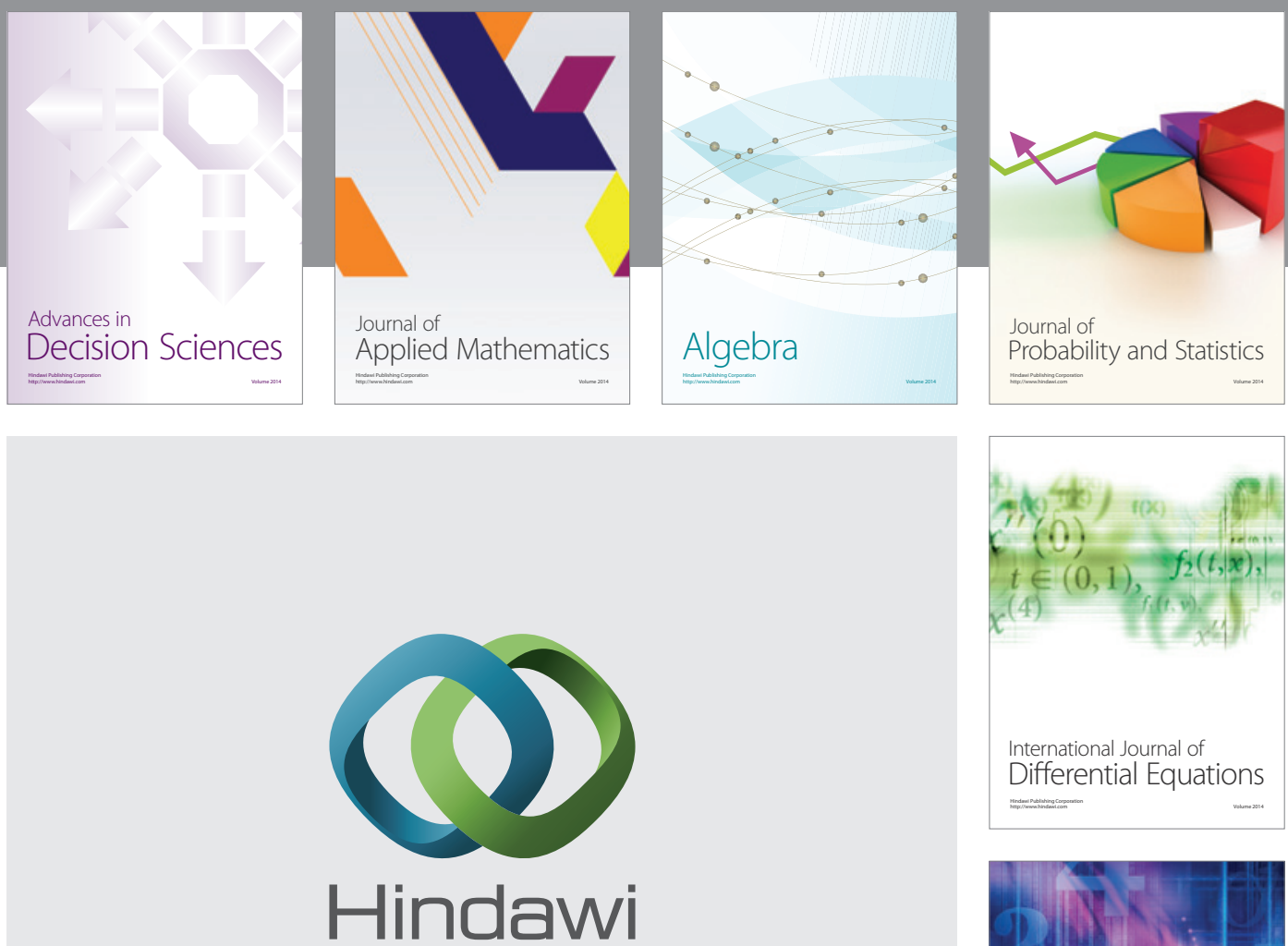

Submit your manuscripts at http://www.hindawi.com
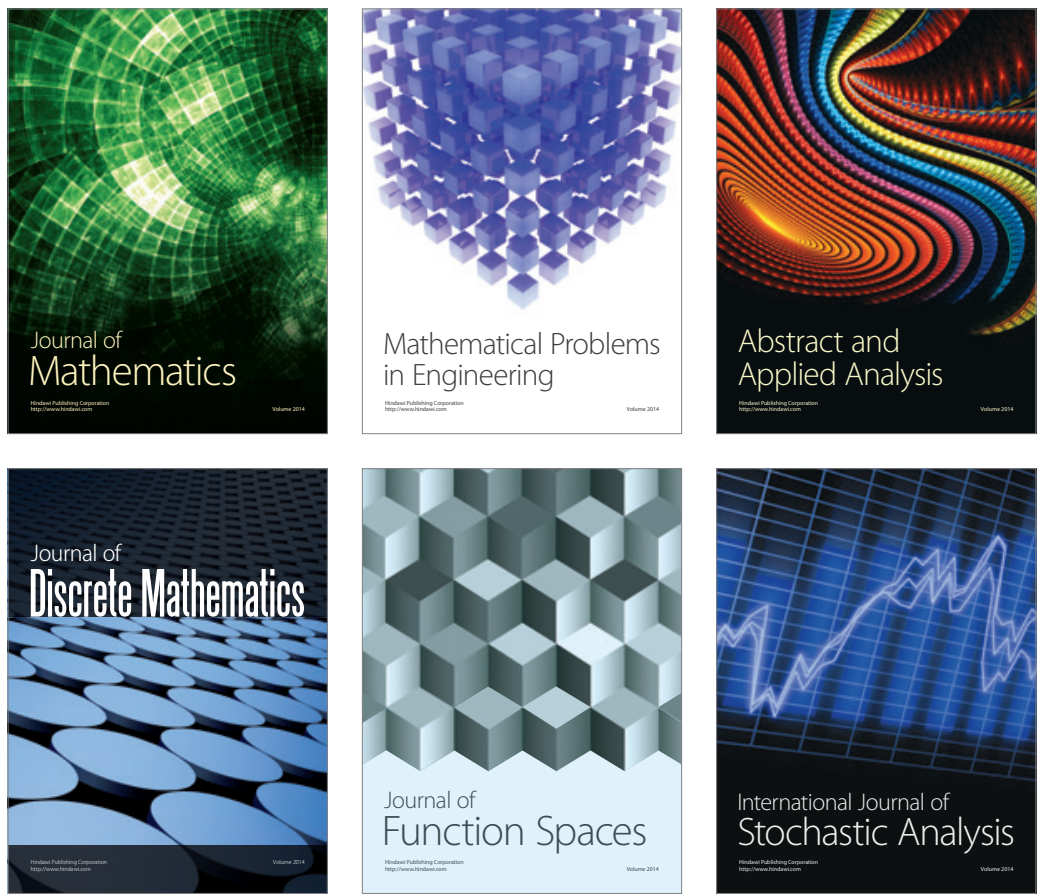

Journal of

Function Spaces

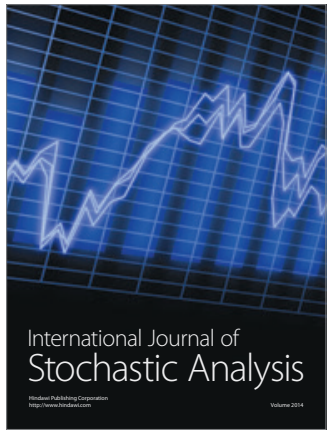

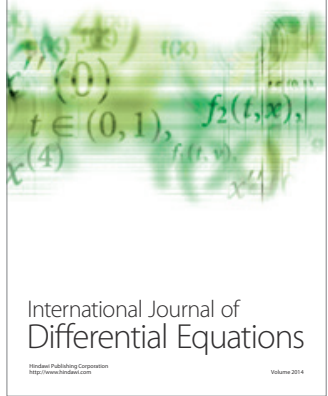
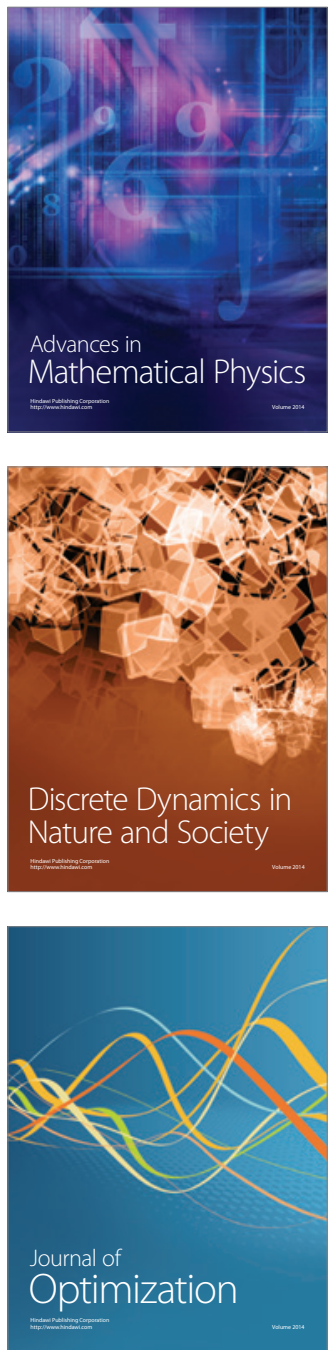